inflammatory markers (C-reactive protein (CRP), tumor necrosis factor (TNF), interleukin (IL)-6). Venous blood samples of glucose and insulin were taken while fasting and during OGTT. Insulin resistance was estimated with the fasting homeostasis model assessment (HOMA) index.

Results: FM, FFM and VF were not different between groups. Diabetes was diagnosed in two subjects in both groups ( $2 \mathrm{hr}$ post OGTT glucose $\geq 11.1 \mathrm{mmol} / \mathrm{l}$ ). Four COPD and $1 \mathrm{C}$ had impaired fasting glucose (fasting glucose 5.6-6.9 mmol/l) while $1 \mathrm{COPD}$ and 2 $\mathrm{C}$ had impaired glucose tolerance (2hr post OGTT glucose 7.8-11.1 mmol/l). In COPD patients a negative correlation was found between the HOMA index and $\mathrm{FEV}_{1}\left(\mathrm{r}^{2}: 0.52, P<0.05\right)$.

Conclusions: COPD subjects with high waist circumference are similar to control subjects in term of FFM and FM, level of systemic inflammation and response to OGTT. In COPD, the severity of the disease is associated with an insulin resistance that may potentiate the risk for the development of type 2 diabetes in these patients.

\section{Assessment of Two Training Modalities Effects on Exercise Tolerance after a Cardiac Rehabilitation Program and 3 Months Later in Off-Pump Coro- nary Artery Bypass Patients}

Kervio G., N.S. Ville, S. Bourdet, Centre d'Innovation Technologique, Centre Cardio-Pneumologique, CHU Pontchaillou, 35033 Rennes, France

C. Le Breton, Clinique Saint-Yves, 4 rue Adolphe Leray, 35000 Rennes, France

F. Carré, Centre d'Innovation Technologique, Centre Cardio-Pneumologique, CHU Pontchaillou, 35033 Rennes, France

Background. Physical training is recommended as an efficient therapy in patients with coronary disease. However its effects should depend on its modalities. The aim of this study was to compare the effects of continuous and interval training after off-pump coronary artery bypass surgery (CABG).

Methods. Twenty-three male patients $(59.5 \pm 1.2 \mathrm{yr})$ underwent a randomized physical training using con- tinuous $(\mathrm{C}, \mathrm{n}=10)$ or interval $(\mathrm{IT}, \mathrm{n}=13)$ modalities, over 3 weeks ( 5 h. a week). All patients performed a symptom-limited exercise test with measurements of ventilatory threshold (VT) and peak workload (WL), oxygen uptake $\left(\mathrm{VO}_{2}\right)$ and heart rate $(\mathrm{HR})$, before, at the end and 3 months after the training period. They also completed the SF-36 questionnaire to assess their quality of life.

Results. Both $\mathrm{C}$ and IT improved $\mathrm{WL}$ and $\mathrm{VO}_{2}$ at VT $(P<0.05)$ and at peak exercise $(P<0.05)$. Only IT increased peak HR and HR reserve $(P<0.01)$. In both groups quality of life was improved $(P<0.05)$. In both groups, compared to after training, the 3-months assessment showed an increase in peak and ventilatory threshold WL, and in peak HR $(P<0.05)$ without any change in peak $\mathrm{VO}_{2}$ and VT HR. No difference was observed between groups whatever the period studied. Back to home, in accordance with the given recommendations, most of the patients carried on their physical training over 3 months. They performed either outside walking $(n=9)$ or cycling sessions $(n=1)$ or both activities $(n=11)$. No difference concerning the global training schedule was noted regardless their group $\left(5.4 \pm 1.2 v s .6 .0 \pm 1.1\right.$ h.week $^{-1}$ for C and IT, respectively).

Conclusions. In off-pump CABG patients, similar improvements of exercise capacity and quality of life perception were observed after both $\mathrm{C}$ and IT programs. Only peak HR was increased after interval training. Thus, IT is a good alternative to $\mathrm{C}$ training in this population. Aerobic fitness still remained stable or increased after 3 months of individual practice which duration exceeded the minimal values recommended.

\section{The Effect of Pulmonary Rehabilitation on Pedometer-Measured Physical Activity}
McCusker Corliss, RN, BS and Richard ZuWallack, $M D$, The Northeast Pulmonary Rehabilitation Con- sortium, Hartford, CT, USA.

Background: Pulmonary rehabilitation results in favorable outcomes across multiple areas, including exercise capacity, dyspnea, and health status. The effectiveness of this intervention on increasing physical 
activity levels, however, has received less attention. Accordingly, we tested the potential usefulness of a commercially-available pedometer (NL-2000) as an outcome measure for pulmonary rehabilitation. This instrument, which is a uniaxial accelerometer, accurately detects walking activity in healthy adults.

Methods: Fifty-five patients with COPD who were referred to participating centers in the Northeast Pulmonary Rehabilitation Consortium (mean age 69 $\mathrm{yr}, \mathrm{FEV}_{1} 45 \%$ ) were studied after informed consent was obtained. All were given usual outpatient pulmonary rehabilitation, with pre- to postrehabilitation assessments of the six minute walk distance (6MWD), MRC dyspnea, and health status, using a self-administered version of the Chronic Respiratory Disease Questionnaire (CRQ). Pedometer activity was measured over a $\sim$ one-week period early in rehabilitation and again for $\sim 1$ week at the end of rehabilitation. The device was not worn during rehabilitation sessions. Patients recorded in a diary the exact times they wore the device. Pre- to postrehabilitation changes in NL-2000 counts per hour worn $\left(\mathrm{NL} \cdot \mathrm{hr}^{-1}\right)$ were evaluated, as well as the standard outcome variables (6 MWD, MRC, and CRQ).

Results: Improvements were observed in the 6MWD (45 m.), MRC dyspnea (- 0.54 units) and the CRQ total score (10 units) (all, $P<0.001)$. However, there was no change in $\mathrm{NL} \cdot \mathrm{hr}^{-1}(30$ counts, $\mathrm{I} P$. $=0.11)$. $\mathrm{NL} \cdot \mathrm{hr}^{-1}$ were lower on rehabilitation days than on nonrehabilitation days; after adding this as a covariate, changes in activity became statistically significant.

Conclusion: These results suggest that either pedometer activity assessment is relatively insensitive to detecting activity in slow-moving patients with COPD and/or pulmonary rehabilitation has less effect on increasing activity than in improving exercise performance, dyspnea, or health status.

\section{Is Prolonged and Repeated Exercise-Induced Myo- cardial Ischemic Training Deleterious?}

\author{
Noël Martin, Jean Jobin, Audrey Marcoux, Luce \\ Boyer, Gilles R Dagenais, Peter Bogaty, Institut \\ universitaire de cardiologie et de pneumologie, \\ Hôpital Laval, Québec, Qc, Canada
}

Background: In patients with ischemic heart disease (IHD), the current guidelines on exercise prescription recommend that exercise training intensity be 10 beats/min below the heart rate at which there is $>1$ mm ST-segment depression (ischemic threshold). However, it is not well established that exercise training above the ischemic threshold is harmful.

Methods: Twenty-two patients with angiographically documented IHD ( $>70 \%$ stenosis) were randomized to exercise training either at a target intensity that induced myocardial ischemia (ischemic group) or that adhered to current guidelines (control group). Training was progressively increased to 60 min under continuous ECG monitoring. Cardiac troponin T (cTnT) was measured at regular intervals. Ambulatory ECG monitoring was performed before and after 6 wkof training and left ventricular function was evaluated by echocardiography in the ischemic group after at least $6 \mathrm{wk}$ of training.

Results: The ischemic training sessions were very well tolerated. The ischemic group had myocardial ischemia during the first 20, 40, and $60 \mathrm{~min}$ exercise sessions for $12.3 \pm 6.8 \mathrm{~min}, 29.0 \pm 12.9 \mathrm{~min}$ and 49.8 $\pm 2.2 \mathrm{~min}$, respectively, with ST-segment depression ranging from 1.0 to $2.1 \mathrm{~mm}$. The estimated myocardial work (as expressed by RPP) during the training session was also higher in the ischemic than in the control group for the first $20 \mathrm{~min}$ (17 $354 \pm 6528$ vs $13355 \pm 2936$ beats/min $\bullet \mathrm{mmHg}$, respectively; $\mathrm{P}=0.08), 40 \min (16329 \pm 5407$ vs $12452 \pm 2330$ beats/min $\bullet \mathrm{mmHg}$, respectively; $\mathrm{P}=0.04)$, and $60 \mathrm{~min}$ training sessions $(18750 \pm 5698$ vs $13352 \pm 2947$ beats/min $\bullet \mathrm{mmHg}$, respectively; $\mathrm{P}=0.02$ ) No patient in either group demonstrated significant arrhythmias nor increased cTnT. The measured cTnT stayed below the detectable values of the essay $(>0.01 \mu \mathrm{g} / \mathrm{l})$ for 